\title{
Path topology dependence of adiabatic time evolution
}

\author{
Atushi Tanaka ${ }^{1, *}$ and Taksu Cheon ${ }^{2, \dagger}$ \\ ${ }^{1}$ Department of Physics, Tokyo Metropolitan University, Hachioji, Tokyo 192-0397, Japan \\ ${ }^{2}$ Laboratory of Physics, Kochi University of Technology, Tosa Yamada, Kochi 782-8502, Japan
}

\begin{abstract}
An adiabatic time evolution of a closed quantum system connects eigenspaces of initial and final Hermitian Hamiltonians for slowly driven systems, or, unitary Floquet operators for slowly modulated driven systems. We show that the connection of eigenspaces depends on a topological property of the adiabatic paths for given initial and final points. An example in slowly modulated periodically driven systems is shown. These analysis are based on the topological analysis of the exotic quantum holonomy in adiabatic closed paths.
\end{abstract}

PACS numbers: 03.65.-w,03.65.Vf,02.40.Pc

\section{INTRODUCTION}

The adiabatic theorem for isolated quantum systems is a basic principle of the quantum dynamics: Once the system is prepared to be in a stationary state, the system remains to be stationary as long as the parameters of the system are varied slow enough. There are many proof for slowly driven systems [1-3], which are described by Hermitian Hamiltonian, as well as slowly modulated driven systems [4-6], which are described by unitary Floquet operators. The adiabatic theorem has diverse applications, e.g. in molecular science and solid state physics $[7,8]$, quantum holonomy [9-11] and adiabatic quantum computation [12-14].

In this article, we examine how the final stationary state of the adiabatic time evolution depends on the path in the adiabatic parameter space. In particular, we here focus on the eigenspaces corresponding to the initial and final stationary states, and we will ignore the phase information in the following. First, we will show that the final stationary state generally depends on the adiabatic path, although the initial adiabatic parameter and initial stationary state are kept fixed. It turns out that the topology of the adiabatic path plays the key role there. Second, we will show that the discrepancy between two final stationary states corresponding to two different adiabatic path is characterized by a permutation matrix, which is governed by a homotopy equivalence. Our idea is an application of the topological formulation for the exotic quantum holonomy $[15,16]$, which concerns the nontrivial change in eigenspaces induced by adiabatic cycles [17].

The present argument heavily relies on topology, in particular the concept of homotopy and its application to the covering map. At the same time, our argument is formal in the sense of mathematics. Since our argument relies only on an elementally account of homotopy and covering map, we refer textbooks of topology for more mathematical description [18-20]. The covering

\footnotetext{
* http://researchmap.jp/tanaka-atushi/

$\dagger$ http://researchmap.jp/T_Zen
}

map is also discussed in a study of phase holonomy of non-Hermitian quantum systems [21].

The plan of this article is the following. In Section 2, we introduce the lifting of adiabatic paths for our problem. This is considered to be an extension of the lifting for the phase holonomy [22, 23]. In Section 3, we present the main results. An example is shown in Section 4. We conclude this article in Section 5.

\section{LIFTING ADIABATIC PATHS}

The lifting of adiabatic paths is the central concept for the theory of conventional quantum holonomy [9-11]. We take over this concept to examine the path-dependence of eigenspaces. In this sense, our approach is a straightforward extension of the works by Simon [22] and Aharonov and Anandan [23].

We here focus on the simplest case where the system is described by a $N$-level Hermitian Hamiltonian $H(\lambda)$ with an adiabatic parameter $\lambda$, whose space is denoted as $\mathcal{M}$. The energy spectrum of $H(\lambda)$ is assumed to be discrete and non-degenerate for an arbitrary $\lambda$ in $\mathcal{M}$. Let $P_{n}(\lambda)$ be the eigenprojector corresponding to an eigenenergy $E_{n}(\lambda)(n=0,1, \ldots, N-1)$. Our assumption on the eigenenergies ensures that $E_{n}(\lambda)$ and $P_{n}(\lambda)$ are smooth in $\mathcal{M}$. Also, $P_{n}(\lambda)$ is rank-one. We remark that it is straightforward to extend the following analysis to unitary Floquet systems, if the spectrum of the Floquet operator is discrete and non-degenerate.

We examine all eigenprojectors at a time, which facilitates to compare the changes in eigenspaces induced by two adiabatic paths. From the eigenprojectors $P_{n}(\lambda)$ for a given point $\lambda$ in $\mathcal{M}$, we introduce an ordered set of the eigenprojectors

$$
p(\lambda) \equiv\left(P_{0}(\lambda), P_{1}(\lambda), \ldots, P_{N-1}(\lambda)\right) .
$$

We may introduce another ordered sets, since the order is arbitrary. Let $\sigma$ denote a permutation of quantum numbers $0,1, \ldots, N-1$. In other words, $\sigma$ is an element of $N$-th symmetric group $\mathcal{S}_{N}$. Let $p_{\sigma}(\lambda)=\sigma(p(\lambda))$, i.e.,

$$
p_{\sigma}(\lambda) \equiv\left(P_{\sigma(0)}(\lambda), P_{\sigma(1)}(\lambda), \ldots, P_{\sigma(N-1)}(\lambda)\right),
$$


where $\sigma(n)$ is the permutated quantum number for a given quantum number $n$.

We introduce a fiber at $\lambda$ in $\mathcal{M}$ :

$$
F_{\lambda} \equiv \bigcup_{\sigma \in \mathcal{S}_{N}}\left\{p_{\sigma}(\lambda)\right\}
$$

For an arbitrary pair of elements, say $p_{\sigma^{\prime}}(\lambda)$ and $p_{\sigma^{\prime \prime}}(\lambda)$, of $F_{\lambda}$, there is a unique permutation $\sigma \in \mathcal{S}_{N}$ that satisfies $p_{\sigma^{\prime \prime}}(\lambda)=\sigma\left(p_{\sigma^{\prime}}(\lambda)\right)$, i.e., $\sigma^{\prime \prime}(n)=\sigma\left(\sigma^{\prime}(n)\right)$ for an arbitrary $n$. In this sense, we call $\mathcal{S}_{N}$ a structural group of the fiber $F_{\lambda}$.

A total space $\mathcal{P}$ consists of the fibers $F_{\lambda}$ in $\mathcal{M}$ :

$$
\mathcal{P} \equiv \bigcup_{\lambda \in \mathcal{M}} F_{\lambda}
$$

which naturally accompanies a projection

$$
\pi: \mathcal{P} \rightarrow \mathcal{M}
$$

by construction. Hence we obtain a fiber bundle consists of the total space $\mathcal{P}$, the projection $\pi$, the base manifold $\mathcal{M}$ and the fiber $F_{\lambda}$.

Utilizing the fiber bundle introduced above, we introduce a lifting of a path $C$ in $\mathcal{M}$ to $\mathcal{P}$ in order to examine the adiabatic time evolution of $p$ along $C$. Let $\lambda_{\mathrm{i}}$ and $\lambda_{\mathrm{f}}$ denote the initial and final points of $C$, respectively.

The adiabatic time evolution of $p \in \mathcal{P}$ can be determined by the time evolution of each eigenprojector $P_{n}$. The adiabatic theorem ensures that the final state of the adiabatic time evolution along $C$ is unique for a given initial stationary state $P_{n}\left(\lambda_{\mathrm{i}}\right)$. Accordingly the initial $p_{\mathrm{i}} \in F_{\lambda_{\mathrm{i}}}$ and the adiabatic path $C$ uniquely determines the final point $p_{\mathrm{f}} \in F_{\lambda_{\mathrm{f}}}$. Let $\phi_{C}$ from $F_{\lambda_{\mathrm{i}}}$ to $F_{\lambda_{\mathrm{f}}}$ denote the mapping from the initial to the final point, i.e.,

$$
p_{\mathrm{f}}=\phi_{C}\left(p_{\mathrm{i}}\right)
$$

Namely, the mapping $\phi_{C}$ describes the change of $p$ induced by the adiabatic path $C$.

The projection $\pi$ introduced in Eq. (5) satisfies the axiom of covering projections [18]. Namely, for a given point $\lambda$ in $\mathcal{M}$, there is an open subset $U$ of $\mathcal{M}$ that satisfies the following: $\pi^{-1}(U)$ is a disjoint union of connected open subset of $\mathcal{P}$. Each of the disjoint component $U_{j}$ is mapped homeomorphically onto $U$ (Fig. 2). The covering map structure determines a various properties of $\phi_{C}$. In particular, it will be shown below that the homotopic classification of the paths plays the central role here.

If $C$ is a closed path with a given initial point $\lambda_{\mathrm{i}}$, the mapping $\phi_{C}$ on $F_{\lambda_{\mathrm{i}}}$ is called the monodromy action (Theorem 11.22 in Ref. [18]). We also note that $\phi_{C}$ can be regarded as a permutation of eigenprojector at $\lambda=\lambda_{\mathrm{i}}$. Since our argument in the next section much owes to the properties of the monodromy action (e.g. shown in Ref. [18]), we will quote the relevant result where appropriate.

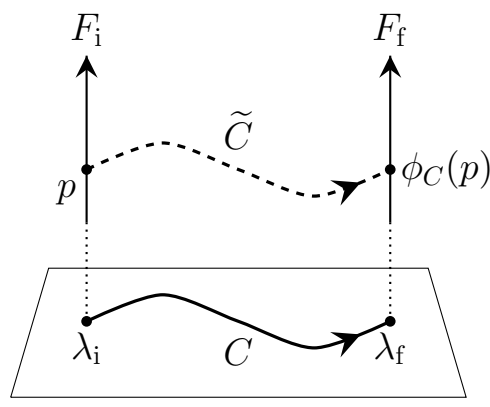

FIG. 1. Lifting adiabatic path $C$ in $\mathcal{M}$ to $\mathcal{P}$ (Eq. (4)), which is made of fibers $F_{\lambda}$ (Eq. (3)). Let $\lambda_{\mathrm{i}}$ and $\lambda_{\mathrm{f}}$ denote the initial and final point of $C$, respectively. The lifted path $\tilde{C}$ starts from $p$, which is in the initial fiber $F_{\mathrm{i}}$, and satisfies the adiabatic Schrödinger equation for the ordered set of eigenprojectors. We introduce the mapping $\phi_{C}$ from $F_{\text {i }}$ to the final fiber $F_{\mathrm{f}}$, so that $\phi_{C}(p)$ is the final point of $\tilde{C}$.

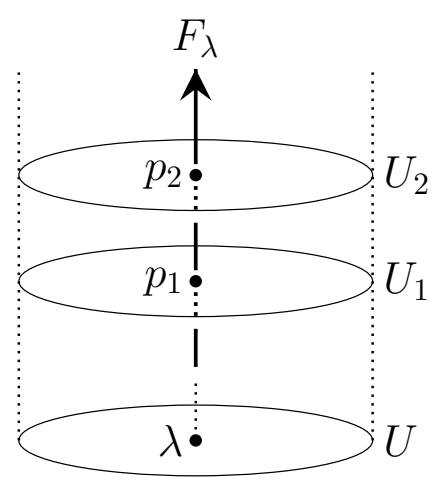

FIG. 2. A schematic picture of the covering map $\pi: \mathcal{P} \rightarrow \mathcal{M}$ (Eq. (5)). Let $\lambda$ be a point in an open set $U \subset \mathcal{M}$. Points $p_{j}$ in the fiber $F_{\lambda}$ (Eq. (3)) satisfies $\pi\left(p_{j}\right)=\lambda$. When $\pi$ is a covering map, $\pi^{-1}(U)$ consists of disjoint union of open sets $U_{j}$, each of which is mapped homeomorphically onto $U$.

\section{COMPARISON OF ADIABATIC PATHS}

We lay out our main results in this section. We compare two adiabatic paths $C_{1}$ and $C_{2}$, which have the same initial and final points $\lambda_{\mathrm{i}}$ and $\lambda_{\mathrm{f}}$, in the adiabatic parameter space $\mathcal{M}$. For a given initial eigenprojector at $\lambda_{\mathrm{i}}$, we will elucidate how the eigenprojectors at the $\lambda_{\mathrm{f}}$ depends on $C_{1}$ and $C_{2}$, by examining the adiabatic time evolutions of the ordered set of eigenprojectors (Eq. (1)). In other words, we examine how $\phi_{C}$, which is a mapping from $F_{\lambda_{\mathrm{i}}}$ to $F_{\lambda_{\mathrm{f}}}$, depends on the topology of the path.

First of all, we examine the case that $C_{1}$ is homotopic to $C_{2}$, which is denoted as $C_{1} \sim C_{2}$. Namely, we suppose that we may smoothly deform $C_{1}$ to $C_{2}$, while keeping its initial and final points. We remark that this is the case where most conventional analyses of the periodic adiabatic time evolution have focused on.

If $C_{1}$ and $C_{2}$ are homotopic, $\phi_{C_{1}}$ and $\phi_{C_{2}}$ are identical, due to the homotopy lifting property (e.g., Theorem 
11.13 in Ref. [18]). Hence, an arbitrary initial eigenprojector is adiabatically transported to the same final point through the paths $C_{1}$ and $C_{2}$.

Utilizing this result, we denote by $\phi_{[C]}$, instead of $\phi_{C}$, where $[C]$ denotes the equivalence class of a path $C$ under the homotopic classification.

Secondly, we proceed to the case where $C_{1}$ is not homotopic to $C_{2}$. We compare these paths with a closed path $C \equiv C_{1} \cdot\left(C_{2}\right)^{-1}$, where the inverse path of $C_{2}$ follows after $C_{1}$. Hence the initial point of $C$ is $\lambda_{\mathrm{i}}$. If a closed path $\gamma$, whose initial point is $\lambda_{\mathrm{i}}$, in $\mathcal{M}$ is homotopic to $C$, the following formula for $\phi_{[C]}$ holds:

$$
\left(\phi_{\left[C_{2}\right]}\right)^{-1} \circ \phi_{\left[C_{1}\right]}=\phi_{[\gamma]}
$$

where $\circ$ denotes the composition of the mappings $\phi_{C}$. Eq. (7) is shown in the following way. Because of $C \sim \gamma$, $\phi_{[C]}=\phi_{[\gamma]}$ holds. On the other hand, $\phi_{[C]}=\left(\phi_{\left[C_{2}\right]}\right)^{-1} \circ$ $\phi_{\left[C_{1}\right]}$ holds from the definition of $\phi_{\left[C_{1} \cdot\left(C_{2}\right)^{-1}\right]}$ (Eq. (6)).

Now our problem, i.e., the comparison of adiabatic time evolutions along adiabatic paths in $\mathcal{M}$, is casted into the analysis of the monodromy action $\phi_{[\gamma]}$ for an arbitrary closed path $\gamma$ in $\mathcal{M}$. We remind that $\phi_{[\gamma]}$ corresponds to a permutation of eigenprojectors induced by the adiabatic time evolution along $\gamma$. For example, if $\gamma$ is contractable to the point $\lambda_{\mathrm{i}}$, which is equivalent to the case $C_{1} \sim C_{2}$ examined above, $\phi_{[\gamma]}$ corresponds to the identical permutation, which implies $\phi_{\left[C_{1}\right]}=\phi_{\left[C_{2}\right]}$.

In order to completely solve our problem, there are two tasks. One is to enumerate all equivalence class $[\gamma]$ of closed paths in $\mathcal{M}$. Namely, we need to identify the first fundamental group $\pi_{1}(\mathcal{M})$ of the adiabatic parameter space $\mathcal{M}$. The other is to examine the monodromy action $\phi_{[\gamma]}$ of eigenspaces, for every $[\gamma]$ in $\pi_{1}(\mathcal{M})$.

There remains a question whether different equivalent classes $[\gamma]$ and $\left[\gamma^{\prime}\right]$ induce different permutations of eigenspaces. In other words, we need to clarify whether $\pi_{1}(\mathcal{M})$ completely characterizes the collection of $\phi_{[\gamma]}$. There are two cases:

1. If $\mathcal{P}$ is simply connected, i.e., $\pi_{1}(\mathcal{P})$ has only a single element, $\phi_{\left[C_{1}\right]}=\phi_{\left[C_{2}\right]}$ holds if and only if $C_{1}$ is homotopically equivalent to $C_{2}$. Hence $\pi_{1}(\mathcal{M})$ offers the complete classification of the adiabatic cycles for our problem.

2. In general, we need to modify the first case above, where the equivalence class of closed paths $\pi_{1}(\mathcal{M})$ is replaced with $H$ where $H \equiv \pi_{1}(\mathcal{M}) / \pi_{*}\left(\pi_{1}(\mathcal{P})\right)$. Namely, $\phi_{\left[C_{1}\right]}=\phi_{\left[C_{2}\right]}$ holds if and only if $C_{1}$ is equivalent to $C_{2}$ under the equivalence class $H$ of closed paths in $\mathcal{M}$.

Here we assume that $\pi$ is a normal covering map, which is equivalent to the condition that $H$ is independent of $p_{\text {i }}$ (Proposition 11.35 in Ref. [18]). As far as we see, this assumption holds in our examples.

These result concern with the group $\Phi$ consists of all possible $\phi_{[\gamma]}$ for an arbitrary closed path $\gamma$. In the theory of covering map, $\Phi$ is called a covering automorphism group, and the above result is just the one-to-one correspondence between $\Phi$ and $H$ (Theorem 12.7 in Ref. [18]).

We examine the latter, general case, where $\mathcal{P}$ is multiply connected. There is a closed path $\tilde{C}$ that is not contractable to a point, in $\mathcal{P}$. We assume that the initial point $p_{\mathrm{i}}$ of $\tilde{C}$ satisfies $\pi\left(p_{\mathrm{i}}\right)=\lambda_{\mathrm{i}}$. Let $C$ be the projection of $\tilde{C}$ into $\mathcal{M}$, i.e., $C \equiv \pi(\tilde{C})$. We note that an arbitrary lift of $C$ to $\mathcal{P}$ is closed. If $C$ is not contractable to a point, i.e., the equivalence class $[C]$ is different from $[e]$, this offers an example of $\phi_{[C]}=\phi_{[e]}$ with $[C] \neq[e]$. Accordingly such $[C]$ makes $H$ nontrivial.

\section{EXAMPLE}

We examine a slowly modulated periodically driven systems in this section. Here a modification of the adiabatic theorem is required for the stationary states that are described by by eigenvectors of a Floquet operator [4$6]$. We choose the periodically driven systems instead of slowly driven Hamiltonian systems because the examples in the latter case requires either the divergence or crossing of eigenenergies, as is seen in the studies of exotic quantum holonomy [15, 24, 25]. We refer Ref. [26] to apply the present formulation for adiabatic paths that involves level crossings.

We compare an arbitrary pair $\left(C_{1}, C_{2}\right)$ of two adiabatic paths in a two level system, where we suppose that the absence of spectral degeneracy in the adiabatic paths. After we lay out our result using a parameterization that is suitable to examine the path topology dependence, of two level systems, we will show an example of nontrivial pair of paths $\left(C_{1}, C_{2}\right)$ using a quantum map.

First, we parameterize the adiabatic path using the set of eigenprojections $P_{1}$ and $P_{2}$

$$
b \equiv\left\{P_{1}, P_{2}\right\},
$$

where the order of the projectors are ignored. Namely, we will specify a point in the base manifold $\mathcal{M}$ by $b$. This amount to the parameterization of adiabatic path by Floquet operator through the spectral decomposition

$$
U=z_{1} P_{1}+z_{2} P_{2},
$$

where $z_{j}$ is $j$-th eigenvalue $(j=1,2)$, since nondegenerate Floquet operator $U$ uniquely specifies $b$. In contrast, there are two possible values of the ordered projector $p$ introduced in Eq. (1), i.e., $\left(P_{1}, P_{2}\right)$ and $\left(P_{2}, P_{1}\right)$. Note that the definition of $b$ in Eq. (8) is straightforward to extend to the systems with an arbitrary number of levels.

Second, we take up a geometric interpretation of $b$ and $p$ for the two level system, utilizing the following parameterization of projection operator

$$
P(\boldsymbol{a})=\frac{1}{2}(1+\boldsymbol{a} \cdot \boldsymbol{\sigma}),
$$




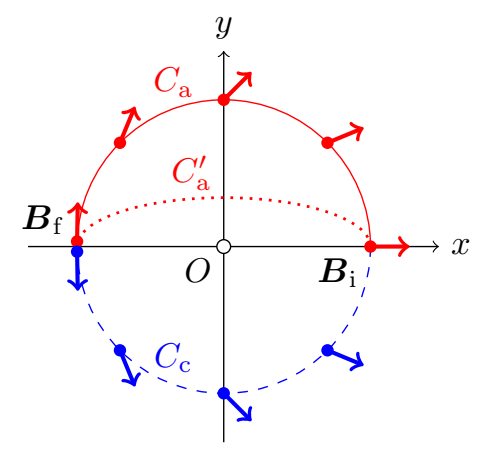

FIG. 3. Adiabatic time evolution of $\boldsymbol{a}$ (thick arrow), which is equivalent to the ordered set of eigenprojectors $p$ for the periodically driven spin- $\frac{1}{2}$ (Eq. (12)). Since $\boldsymbol{a}$ is transported from $\boldsymbol{e}_{x}$ to $\boldsymbol{e}_{y}$ along the adiabatic path $C_{\mathrm{a}}$ (thick curve), the corresponding adiabatic evolution of eigenprojector is from $P\left(\boldsymbol{e}_{x}\right)$ to $P\left(\boldsymbol{e}_{y}\right)$. In other words, the adiabatic mapping of $p$ is $\phi_{\left[C_{\mathrm{a}}\right]}\left(p\left(\boldsymbol{e}_{x}\right)\right)=p\left(\boldsymbol{e}_{y}\right)$, where $p(\boldsymbol{a}) \equiv(P(\boldsymbol{a}), P(-\boldsymbol{a}))$. Other adiabatic path $C_{\mathrm{a}}^{\prime}$ (dotted curve), which is homotopic to $C_{\mathrm{a}}$, provides the same adiabatic mapping, i.e., $\phi_{\left[C_{a}\right]}=\phi_{\left[C_{a}^{\prime}\right]}$. On the other hand, the adiabatic path $C_{\mathrm{c}}$ (dashed curve) is not homotopic to $C_{\text {a }}$ due to an obstacle (a disclination [20,27]) at the origin. The corresponding adiabatic evolution is described as $\phi_{\left[C_{\mathrm{c}}\right]}\left(p\left(\boldsymbol{e}_{x}\right)\right)=p\left(-\boldsymbol{e}_{y}\right)$. We may compare $C_{\mathrm{a}}$ and $C_{\mathrm{c}}$ by a closed path $C=C_{\mathrm{c}}^{-1} \cdot C_{\mathrm{a}}$. The discrepancy between $\phi_{\left[C_{\mathrm{a}}\right]}$ and $\phi_{\left[C_{c}\right]}$ is given by $\phi_{[C]}$, which corresponds to the cyclic permutation of the two items in $p$.

where $\boldsymbol{\sigma}$ is the vector consists of Pauli matrices, and $\boldsymbol{a}$ is a normalized three-dimensional real vector. The eigenprojectors in Eq. (9) can be expressed as $P_{1}=P(\boldsymbol{a})$ and $P_{2}=P(-\boldsymbol{a})$. Now it is straightforward to see that $p$ and $\boldsymbol{a}$ has 1: 1 correspondence, which implies that $\mathcal{P}$ can be identified with $S^{2}$. On the other hand, $\pm \boldsymbol{a}$ correspond to a single point in the $b$-space. Namely, the $b$-space can be regarded as $\mathbb{R} P^{2}$, the real projective plane. Hence the covering map $\pi: S^{2} \rightarrow \mathbb{R} P^{2}$ for the two level system can be regarded as an identification of the antipodal points in the sphere.

Now our argument presented in the previous section is ready to apply. The fundamental class of the base space $\pi_{1}\left(\mathbb{R} P^{2}\right)=\{[e],[\gamma]\}$ has two elements, where $e$ is the closed path that contractable to a point, and the closed path $\gamma$ is not homotopic to $e$. On the other hand, our total space $\mathcal{P}$ is simply connected as $\mathcal{P}=S^{2}$. Hence $[e]$ and $[\gamma]$, the two classes of closed paths, offers two different monodromy map $\phi_{[e]}$ and $\phi_{[\gamma]}$, which correspond to the identity and cyclic permutations of two eigenprojectors, respectively.

We summarize the analysis of two level systems. When the trails of the adiabatic paths $C_{1}$ and $C_{2}$ in $\mathbb{R} P^{2}$ are homotopic, the adiabatic time evolutions of an eigenprojector along $C_{1}$ and $C_{2}$ has no difference. On the other hand, when $C_{1}$ are $C_{2}$ not homotopic, the composite closed path $C_{2}^{-1} \cdot C_{1}$ is homotopic to $\gamma$, and the corresponding discrepancy $\phi_{[\gamma]}=\left(\phi_{\left[C_{2}\right]}\right)^{-1} \circ \phi_{\left[C_{1}\right]}($ Eq. $(7))$ is expressed by the cyclic permutation of two items.

We exemplify the above argument using a slowly modulated driven spin- $\frac{1}{2}$, where we set $\hbar=1$. In the absence of the modulation, our example is described by the following periodically driven Hamiltonian:

$$
H(t)=\frac{1}{2} \boldsymbol{B} \cdot \boldsymbol{\sigma}+\lambda \frac{1-\sigma_{z}}{2} \sum_{m=-\infty}^{\infty} \delta(t-m),
$$

where $\boldsymbol{B}=B\left(\cos \phi \boldsymbol{e}_{x}+\sin \phi \boldsymbol{e}_{y}\right)$ is the static magnetic field confined in $x y$-plane $(B$ and $\phi$ are the cylindrical variables), and $\lambda$ is the strength of the periodic term. The corresponding Floquet operator is, for example

$$
U=e^{-i \lambda\left(1-\sigma_{z}\right) / 2} e^{-i \boldsymbol{B} \cdot \boldsymbol{\sigma} / 2}
$$

which is a quantum map under a rank-1 perturbation [28, 29]. In the following, we examine $U$ under the adiabatic changes of $\boldsymbol{B}$ in $\left(B_{x}, B_{y}\right)$-plane except the origin $\boldsymbol{B}=0$. Also, we set $\lambda=\phi$ along the adiabatic path. Hence $U$ is single-valued in $\left(B_{x}, B_{y}\right)$-plane, since $U$ periodically depends on $\lambda$ with the period $2 \pi$. The eigenvalues of $U$ are, as shown in Ref. [17], $z_{ \pm}=\exp \{-i(\phi \pm \Delta) / 2\}$, where

$$
\Delta=2 \arccos \left(\cos \frac{\phi}{2} \cos \frac{B}{2}\right) .
$$

The corresponding eigenprojectors are $P( \pm \boldsymbol{a})$, where

$$
\begin{aligned}
\boldsymbol{a}=\frac{1}{\sin (\Delta / 2)}[ & \sin \frac{B}{2}\left(\cos \frac{\phi}{2} \boldsymbol{e}_{\rho}-\sin \frac{\phi}{2} \boldsymbol{e}_{\phi}\right) \\
& \left.-\sin \frac{\phi}{2} \cos \frac{B}{2} \boldsymbol{e}_{z}\right],
\end{aligned}
$$

$\boldsymbol{e}_{\rho}=\cos \phi \boldsymbol{e}_{x}+\sin \phi \boldsymbol{e}_{y}$ and $\boldsymbol{e}_{\phi}=-\sin \phi \boldsymbol{e}_{x}+\cos \phi \boldsymbol{e}_{y}$. Note that $\Delta$ and $\boldsymbol{a}$ are not single-valued in $\left(B_{x}, B_{y}\right)$-plane although $U$ is single-valued. We depict adiabatic paths, whose initial and final points in $\left(B_{x}, B_{y}\right)$-plane are $\boldsymbol{B}_{\mathrm{i}} \equiv$ $(\pi, 0)$ and $\boldsymbol{B}_{\mathrm{f}} \equiv(-\pi, 0)$, respectively, and corresponding adiabatic time evolution of eigenspaces in Fig. 3 .

\section{CONCLUSION}

We have shown the path topology dependence of adiabatic time evolution in closed quantum systems through a topological argument, which is based on the recent study on the exotic quantum holonomy [17]. We finally note that examples of systems exhibit non-trivial adiabatic path topology dependence, according to the studies of exotic quantum holonomy in, for example, quantum graphs with generalized connection conditions[3033], many-qubit systems [34], adiabatic quantum computation [14], and the Lieb-Liniger model [35].

\section{ACKNOWLEDGEMENTS}

This research was supported by the Japan Ministry of Education, Culture, Sports, Science and Technology under the Grant number 15K05216. 
[1] M. Born and V. Fock, Z. Phys. A 51, 165 (1928).

[2] T. Kato, J. Phys. Soc. Japan 5, 435 (1950).

[3] J. E. Avron, R. Seiler, and L. Yaffe, Comm. Math. Phys. 110, 33 (1987).

[4] R. H. Young and W. J. Deal Jr., J. Math. Phys. 11, 3298 (1970).

[5] A. Dranov, J. Kellendonk, and R. Seiler, Journal of Mathematical Physics 39, 1340 (1998).

[6] A. Tanaka, Journal of the Physical Society of Japan 80, 125002 (2011).

[7] M. Born and R. Oppenheimer, Annalen der Physik 389, 457 (1927).

[8] M. Born and K. Huang, Dynamical Theory of Crystal Lattices (Oxford University Press, Oxford, 1954).

[9] A. Shapere and F. Wilczek, eds., Geometric phases in physics (World Scientific, Singapore, 1989).

[10] A. Bohm, A. Mostafazadeh, H. Koizumi, Q. Niu, and Z. Zwanziger, The Geometric Phase in Quantum Systems (Springer, Berlin, 2003).

[11] D. Chruściński and A. Jamiołkowski, Geometric Phases in Classical and Quantum Mechanics (Birkhäuser, Boston, 2004).

[12] E. Farhi, J. Goldstone, S. Gutmann, and M. Sipser, "Quantum computation by adiabatic evolution," (2000), arXiv:quant-ph/0001106.

[13] E. Farhi, J. Goldstone, S. Gutmann, J. Lapan, A. Lundgren, and D. Preda, Science 292, 472 (2001).

[14] A. Tanaka and K. Nemoto, Phys. Rev. A 81, 022320 (2010).

[15] T. Cheon, Phys. Lett. A 248, 285 (1998).

[16] A. Tanaka and M. Miyamoto, Phys. Rev. Lett. 98, 160407 (2007).
[17] A. Tanaka and T. Cheon, Phys. Lett. A 379, 1693 (2015).

[18] J. M. Lee, Introduction to Topological Manifolds, 2nd ed., Graduate Texts in Mathematics, Vol. 202 (Springer, New York, 2011).

[19] A. Hatcher, Algebraic Topology (Cambridge University Press, Cambridge, 2001).

[20] M. Nakahara, Geometry, Topology and Physics (IOP Publishing Ltd, Bristol, 1990).

[21] H. Mehri-Dehnavi and A. Mostafazadeh, J. Math. Phys. 49, 082105 (2008).

[22] B. Simon, Phys. Rev. Lett. 51, 2167 (1983).

[23] Y. Aharonov and J. Anandan, Phys. Rev. Lett. 58, 1593 (1987).

[24] T. Cheon, A. Tanaka, and S. W. Kim, Phys. Lett. A 374, 144 (2009).

[25] T. Cheon, A. Tanaka, and O. Turek, Acta Polytechnica 53, 410 (2013).

[26] A. Tanaka and T. Cheon, "A topological formulation for exotic quantum holonomy," (2015), arXiv:1507.02827.

[27] N. D. Mermin, Rev. Mod. Phys. 51, 591 (1979).

[28] M. Combescure, J. Stat. Phys. 59, 679 (1990).

[29] B. Milek and P. Seba, Phys. Rev. A 42, 3213 (1990).

[30] T. Cheon, T. Fülöp, and I. Tsutsui, Ann. Phys. (N.Y.) 294, 1 (2001).

[31] I. Tsutsui, T. Fülöp, and T. Cheon, J. Math. Phys. 42, 5687 (2001).

[32] S. Ohya, Ann. Phys. (NY) 331, 299 (2013).

[33] S. Ohya, Ann. Phys. (NY) 351, 900 (2014).

[34] A. Tanaka, S. W. Kim, and T. Cheon, Europhys. Lett. 96, 10005 (2011).

[35] N. Yonezawa, A. Tanaka, and T. Cheon, Phys. Rev. A 87, 062113 (2013). 